\title{
Wer hat Angst vorm Schurkenstaat? Macht/Raum-Diskurse in Sri Lanka
}

\section{Benedikt Korf, Liverpool}

\section{Macht/Raum-Diskurse und Kritische Geopolitik}

«Kritische Geopolitik» untersucht Prozesse sozialer Raumkonstruktionen im Zusammenspiel von Raum, Politik und Macht. Sie hat wichtige Beiträge dazu geleistet, die Konstruktion von Unterschieden zwischen dem «Selbst» und dem «Anderen» und die dahinter stehenden politischen Interessen zu analysieren und zu zeigen, wie Argumente angeblicher räumlicher Determinismen dabei zum Tragen kommen. Bisher hat sich Kritische Geopolitik insbesondere mit geopolitischen Diskursen und Konstrukten in der internationalen Politik, der globalen Terrorismusdebatte und mit der Legitimität militärischer und politischer Interventionen auseinandergesetzt (z.B. Agnew 2001; DalBY 1991; Dodds \& Sidaway 1994; Ó Tuathail 1996; Ó Tuathail \& Dalby 1998). In diesem Artikel wird der Ansatz der Kritischen Geopolitik genutzt, um politische Diskurse um Ethnizität, Territorialität und Staatlichkeit im derzeitigen Friedensprozess in Sri Lanka zu analysieren.

Zur Anwendung kommt eine geopolitische Diskursanalyse, wie sie von GEARóID Ó TUATHAIL konzeptionalisiert wurde (Ó TuathaIl 2002). Sein Verständnis von Diskurs ist weniger ambitiös als Foucaults philosophisch-historischer Ansatz der Genealogie und Diskursanalyse (Foucault 1972). Ó TUATHAIL hat seine eigene Position als «meso-level discourse analysis» (Ó TuATHAIL 2002) bezeichnet, die sich für die alltägliche Funktion von Diskursen in den öffentlichen politischen Debatten interessiert. Dieser Ansatz verbleibt insofern auf einer realistischen Ebene, als sich hinter Diskursen Akteure mit spezifischen Interessen verbergen und es der geopolitischen Analyse darum geht, die rhetorischen Argumentationslinien der Akteure aufzudecken, durch die Kohärenz und Legitimation in der geopolitischen Praxis erzeugt werden sollen. Die Analyse in diesem Beitrag konzentriert sich darauf, wie Diskurse Handlungen und Strukturen, die eine eigene Ontologie zugesprochen bekommen, objektiv und subjektiv (re-)produzieren (KoRf 2003a). Akteure formen Positionen und rhetorische Argumente und werden gleichzeitig von bereits bestehenden Diskursen und story lines in ihrer Argumentationslinie geprägt (Ó Tuathail 2002). In dieser Form der Diskursanalyse wird also ein handlungstheoretischer Aspekt beibehalten und Diskurs erhält eine schwächere Ontologie als Foucault sie dem Diskurs zubilligt, wenn er vom Ende des Subjekts spricht (Foucault 1972).
Ó Tuathall definiert Diskurs als ein Set von Ressourcen und Fähigkeiten («capabilities»), die uns helfen, die Welt um uns herum zu organisieren und ihr und unseren Handlungen und Praktiken in ihr einen Sinn zu geben. In der geopolitischen Analyse können Diskurse als immaterielle Machtressource aufgefasst werden, die vor allem zwei Zielen dient: zum einen sind Diskurse Instrumente, um kognitive Schemata in einer Gesellschaft und damit die Wahrnehmung der Welt zu formen. Andererseits sind Diskurse ein normatives Mittel, um das «Gute» und das «Böse» zu definieren. Diskurse werden als Ideologie genutzt, wenn sie die Verhandlungsmacht bestimmter Gruppen in der gesellschaftlichen und politischen Arena auf Kosten anderer Gruppen zu untermauern suchen. Ideologien sind also machiavellinische Instrumente zur Herrschaftssicherung. Durch Konstruktionen von Ideologien bieten Diskurse in politischen Auseinandersetzungen einen Anker zur Gruppensolidarisierung.

\section{Schurkenstaaten, «War for Peace» und Terrorismus- diskurse in Sri Lanka}

Zwanzig lange Jahre führten die tamilischen Befreiungstiger und die singhalesisch dominierte Zentralregierung in Sri Lanka einen blutigen Bürgerkrieg. Erst im Februar 2002 wurde zwischen den beiden Kriegsparteien ein Waffenstillstand geschlossen und ein Prozess der Friedensverhandlungen eingeleitet. Dieser Friedensprozess wird jedoch dadurch konterkariert, dass in den Medien und in den öffentlichen Diskursen die territorialen Ansprüche und staatlichen Ambitionen des «ethnisch Anderen» als Aggression gegen das «ethnische Selbst» und die eigene Identität interpretiert werden. Der - reale oder virtuelle - Staat des «Anderen»- mit der Anerkennung seiner «legitimen» Ansprüche auf Territorialität - wird damit zum «Schurkenstaat».

Als «Schurkenstaaten» werden seit einigen Jahren in der internationalen Politik, insbesondere von US-amerikanischen Politikern, diejenigen Staaten gebrandmarkt, die sich nicht durch internationales Recht

Der vorliegende Beitrag ist eine überarbeitete Fassung eines Vortrages, der am 2. Oktober 2003 im AK Politische Geographie am 53. Deutschen Geographentag in Bern gehalten wurde. 
und Verhandlungen von aggressiven Tatbeständen gegenüber ihren Nachbarn abhalten lassen und die eine Bedrohung amerikanischer Sicherheitsinteressen darstellen. Trotz seiner analytischen Unschärfe können vier wichtige Anklagepunkte identifiziert werden: Schurkenstaaten (1) stellen Massenvernichtungswaffen her, (2) unterstützen den weltweiten Terrorismus, (3) unterdrücken ihre eigenen Staatsbürger und (4) drücken ihre Ablehnung gegenüber politischer Einflussnahme durch die USA aus. Schurkenstaaten lassen sich meist nicht von internationalem Recht beeindrukken und sind keine verlässlichen Verhandlungspartner. Sie stellen deshalb eine Bedrohung der Sicherheit der USA und der westlichen Welt dar (KLARE 1995; LiTWAK 2000; RUBin 1999). Es waren insbesondere Noam Chomsky und Jacques Derrida, die sich gegen diese einseitige Weltsicht gestellt haben und sie als Rechtfertigung des Stärkeren zu entlarven suchten (Chomsky 2000; Derrida 2003). Derrida hat argumentiert, dass die Identifizierung von «Schurken» und «Terroristen» dazu dient, tief verwurzelte Ängste und Panik, die mit der globalen Terrorismusbedrohung verbunden werden, zu rationalisieren (DERRIDA 2003).

Die Verbindung von «Schurken»-Argumenten mit einer Strategie des «war for peace» favorisierte lange Zeit auch die srilankische Präsidentin CHANDRIKA BANDaranaike Kumaratunge im Kampf gegen die tamilischen Befreiungstiger im Nordosten Sri Lankas. Schurkenbilder werden von verschiedenen Akteuren im gegenwärtigen Friedensprozess in Sri Lanka aufgegriffen, indem der «ethnisch Andere» als die Ursache für die eigene Misere identifiziert wird - als aggressiver Schurke, der die eigene Identität bedroht. Diese Schurkenbilder werden innerhalb von allen drei wichtigen ethnischen Gruppen in Sri Lanka - Singhalesen, Tamilen und Muslime - für eigene politische Interessen instrumentalisiert. Insbesondere wird die grundlegende Legitimierung der Forderungen nach Staatlichkeit und Verräumlichung ethnischer Identität des «ethnisch Anderen» in Frage gestellt, um die eigene Machtposition sowohl gegenüber den «ethnisch Anderen» also auch innerhalb der eigenen ethnischen Gruppe zu stärken. In letztgenanntem Fall dienen sie der Kohärenz und der Nivellierung von gruppeninternen Widersprüchen. Diese Prozesse der Konstruktion von Schurkenbildern verringern die Bereitschaft zur ethnischen Aussöhnung und verhindern, dass politische Kompromisse mit den «ethnisch Anderen» auf breiter gesellschaftlicher Basis abgestützt werden.

\subsection{Singhalesische Schurkenbilder}

In weiten Teilen der singhalesischen politischen und gesellschaftlichen Elite in Colombo wird die Legitimität der Liberation Tigers of Tamil Eelam (LTTE) mit dem Hinweis darauf in Frage gestellt, dass es sich bei der LTTE um eine terroristische Gruppe handelt. Unter
Bezug auf die internationale Terrorismusdebatte seit dem 11. September hat der Terrorvorwurf die Rolle eines Totschlagarguments erhalten: die srilankische Regierung reiht sich selbst in die Riege der demokratischen Staaten der westlichen Welt im Kampf gegen den Terror ein und spricht gleichzeitig einem zukünftigen Tamilenstaat jede Legitimität $a b$, da er nur ein «Schurkenstaat» sein kann. Auch unter der singhalesischen Diaspora scheint diese Sicht weit verbreitet zu sein. Ein Zitat aus einem Brief der World Association for Peace in Sri Lanka (WAPS), einem Zusammenschluss singhalesischer Diasporaorganisationen, verdeutlicht diese Haltung. Die Diasporaorganisation bittet die Präsidentin, alles in ihrer Macht Stehende zu unternehmen,

«... [to liberate] Sri Lanka from Tamil Tiger terrorists and [to restore] democracy in areas subject to their draconian terror» (INDRAJIT 2003:3).

Der frühere Außenminister und jetzige Präsidentenberater KadiRgamar argumentierte in einer Parlamentsrede, dass Zugeständnisse der Regierung an die LTTE ein «terroristisches Regime» unterstützen und legitimieren und die Integrität des srilankischen Staates gefährden (THE IsLand v. 9.5.2003: 1,3). In der englischsprachigen Presse in Sri Lanka wird vor allem die Zeitung THE IsLAND nicht müde, immer wieder auf die potentielle Untergrabung der Integrität des srilankischen Staates durch die Forderungen der LTTE hinzuweisen (THE IsLaND v. 9.5.2003: 10 und v. 21.5.2003: 8).

Diese Argumentationslinie wurde insbesondere im Mai und Juni 2003 deutlich, als die Sri Lanka Monitoring Mission (SLMM), eine von der norwegischen Regierung entsandte Beobachtergruppe für die Einhaltung des Waffenstillstandes, einen Vorschlag unterbreitete, um die zunehmenden militärischen Zusammenstöße zwischen der srilankischen Marine und den sogenannten «Sea Tigers», der Marine der LTTE, zu reduzieren. Der Vorschlag, den Sea Tigers einen Status einzuräumen, der der srilankischen Marine nahe kommt, und ihr quasi-souveräne maritime Zonen zuzugestehen, führte zu einem Aufschrei in der Zeitung THE ISLAND und unter nationalistischen politischen Akteuren in Colombo: Die Sea Tigers, eine «terroristische Militärorganisation», mit der srilankischen Marine, die ein legitimes Instrument des souveränen Staates Sri Lanka sei,zu vergleichen, wurde scharf angegriffen und der SLMM Parteilichkeit zugunsten der LTTE unterstellt (z.B. The IsLand v. 6.5.2003: 10). In der Zeitung THE IsLAND v.13.6.2003: 10 heißt es dazu:

«Pro-LTTE journals have been at pains to complain about recruitment by the government to the armed forces as well as purchase of military equipment, forgetting that the armed forces of a nation have to be in a state of readiness to meet internal and external threats and it cannot be compared with clandestine forces of a terrorist organisation.» 
In der argumentativen Untermauerung des Schurkenstaatsvorwurfs wird auch auf vermeintliche Menschenrechtsverletzungen der LTTE hingewiesen. Ungefähr zeitgleich mit der Kritik an der norwegischen SLMM veröffentlichte THE IsLand ein Dossier der angesehenen tamilischen Menschenrechtsorganisation University Teachers for Human Rights - Jaffna (UTHR-J) über die Einschüchterung und Ermordung tamilischer Oppositioneller durch die LTTE. Damit sollte die Legitimierung der LTTE als Vertretung der tamilischen Bevölkerung weiter untergraben werden: Die LTTE sei im Prozess, einen «Schurkenstaat» im Nordosten zu etablieren, ihre politischen Gegner zu eliminieren und die staatliche Integrität Sri Lankas in Frage zu stellen (obwohl sie sich öffentlich in den Verhandlungen zu einer föderalen Lösung innerhalb des srilankischen Staates bekannt hat). Zur Unterstreichung dieses Arguments wird immer wieder vorgebracht, es sei schließlich die LTTE gewesen, die in früheren Friedensverhandlungen zu Kampfhandlungen zurückgekehrt sei $(1990,1995)$ und damit ihre Unberechenbarkeit als Terrororganisation unter Beweis gestellt habe.

\subsection{Tamilische Schurkenbilder}

In Diskussionen unter Tamilen wird der srilankische Staat oft als «Schurke» dargestellt, der der tamilischen Minderheit gleiche Rechte verweigert hat und sie diskriminiert. Seit der Eskalation des ethnisierten Konfliktes dominieren Polizei und Militär, die fast ausschließlich Singhalesen rekrutieren, in den Gebieten den öffentlichen Raum im Nordosten, die nicht von der LTTE kontrolliert werden. Die Armee etablierte ein System von Checkpoints mit täglichen Kontrollen der Zivilbevölkerung. Dies eröffnet täglichen Schikanen Tür und Tor und führt zu einem System der Unberechenbarkeit. Rechtsstaatliche Prinzipien sind in den Bürgerkriegsgebieten weitgehend außer Kraft gesetzt, auch aufgrund des Ausnahmezustandes. Das Militär erscheint vielen Tamilen als fremde Besatzungsarmee, nicht jedoch als legitime Vertretung des srilankischen Staates.

Unter dem Schutz von Polizei und Armee konnten Singhalesen im Nordosten illegale Aktivitäten durchführen, z.B. verlassenen Besitz übernehmen, Fischereigründe ausbeuten, zu denen tamilischen Fischern der Zugang verwehrt wurde (Rösel 1997: 347). Dies erscheint in den Augen vieler Tamilen als eine Fortführung der staatlichen Diskriminierung, die sich in den 1960er und 1970er Jahren in der Sprachen- und Bildungspolitik widerspiegelte (RösEL 1997: 102-108; TAMBIAH 1986). Der Bürgerkrieg führte außerdem zu einem ökonomischen Niedergang der Kriegsgebiete, viele wohlhabende Tamilen wanderten aus, so dass es zu einem brain drain gekommen ist. Die Tamilen sehen sich als die Hauptleittragenden des Krieges. Im
Alltag drohten ihnen Verhaftungen, Enteignungen und Morde und das umfassende Kontrollsystem erschwerte ihnen die tägliche Lebensführung und stellte sie unter einen Generalverdacht. Dieses Vorgehen des Staates unterhöhlte die Würde der tamilischen Bevölkerung und führte zu einer weiteren Distanzierung zwischen Staat und tamilischen Bürgern.

Der «Schurke», der hier konstruiert wird, ist der srilankische Staat und dessen politisch-administratives System. Dieser «Schurkenstaat» versucht, die Integrität des Tamil Eelam mit seiner ethnisch einseitigen Besiedlungsstrategie und einer ethnisch diskriminierenden Politik zu unterminieren. Die Versuche der LTTE, quasi-staatliche Strukturen im Nordosten zu etablieren, werden deshalb von vielen Tamilen toleriert, wenn nicht sogar gutgeheißen, denn sie erscheinen ihnen legitim in der Bildung eines tamilischen Homelands, das sich abgrenzt vom singhalesischen «Schurkenstaat». Auch wenn die LTTE in den laufenden Friedensverhandlungen einer föderalen Lösung innerhalb des srilankischen Staates zugestimmt hat, so wird doch auf tamilischer Seite erwartet, dass der Nordosten eine weitreichende Autonomie zugestanden bekommt, als Sicherheitsgarantie gegen mögliche zukünftige Aggressionen des singhalesischen Staates gegenüber der tamilischen Bevölkerung. Die LTTE scheint in der Zwischenzeit "Fakten» schaffen zu wollen, indem sie im von ihr kontrollierten Wanni quasi-staatliche Strukturen etabliert (Justizsystem, Zoll, Polizei), die hoheitliche Aufgaben wahrnehmen (vgl. University TeACHERS For Human Rights - JafFNa (UTHR - J) 2003). Berichte darüber nähren wiederum die Schurkendiskurse in der singhalesischen Presse.

\subsection{Muslimische Schurkenbilder}

Muslimische Politiker versuchen in jüngster Zeit verstärkt, die muslimische Frage im Osten Sri Lankas auf die politische Agenda zu bringen. Viele Muslime im Osten stehen einer weitgehenden Autonomie des Nordostens unter Federführung der LTTE reserviert gegenüber, denn sie befürchten, als Minderheit in einem von der LTTE dominierten Nordoststaat diskriminiert zu werden. Die Muslime sehen Ansätze eines LTTE-Schurkenstaates bereits als gegeben an, der sich in der Besteuerung der Muslime durch die LTTE und in dem von der LTTE oktroyierten Gerichtswesen zeigt.

Die tamilisch-muslimischen Beziehungen leiden traditionell unter sowohl konkurrierenden Ansprüchen an Landrechten als auch ethnisch dominierten Handelsstrukturen (McGilvray 2003; SchriJvers 1997). Ein großer Teil des muslimischen Landbesitzes im Osten Sri Lankas, insbesondere in Batticaloa, liegt in den von der LTTE kontrollierten Gebieten und ist seit dem Waffenstillstand von der LTTE erst teilweise an die 
muslimischen Besitzer zurückgegeben worden. Frustration und Antagonismen zwischen den beiden Gruppen scheinen sich soweit aufgestaut zu haben, dass sie schon bei kleinen Anlässen aktiviert werden und kollektive Betroffenheit und Aggressivität gegenüber den «ethnisch Anderen» erzeugen können. In den 1990er Jahren kam es zu vielen gewalttätigen Auseinandersetzungen zwischen Muslimen und Tamilen an der Ostküste (GoodHAND \& Lewer 1999: 73-79), und seit dem Waffenstillstand hat sich diese Gegend (vor allem Muttur, Kinniya, Trcinomalee) als besonders instabil erwiesen. Zusammenstöße entladen sich oft an relativ kleinen Streitigkeiten, zeigen jedoch das angestaute Aggressionspotential auf, das sich gegen den «ethnisch Anderen» als Schurken und Ursache des eigenen Leidens richtet.

\section{Virtuelle Schurkenstaaten und Fiktionen der Reinheit}

In Tabelle 1 werden die drei Diskursmuster zusammengefasst und dahinter stehende, strategische Interessen dargelegt. Die Analyse der politischen Interessen derjenigen Akteure, die die Schurkendiskurse aktiv in die jeweilige «eigene» Gesellschaft transportieren, zeigt, dass Schurkenbilder einer Strategie dienen, welche bestehende soziale, politische und ökonomische Strukturbrüche innerhalb der eigenen Gruppe überdecken soll. Dies geschieht dadurch, dass die Schuld beim «Anderen», dem Schurken und seiner territorialisierten Form des aktuellen oder potentiellen «Schurkenstaates», gesucht - und gefunden - wird. Diese Analyse zeigt auch sehr deutlich, wie die politischen Diskurse entlang «ethnischer» Logiken geführt werden.

\subsection{Ausflüchte aus der eigenen Krise}

Im singhalesischen Diskurs wird die LTTE als pure Terrororganisation (ohne legitime politische Basis) «entlarvt» und ihre Bemühungen, eigene Staatlichkeit im Nordosten zu schaffen, als Angriff auf die Integrität des srilankischen Staates gebrandmarkt. Ein nachhaltiger und demokratischer Ausgleich mit den anderen ethnischen Gruppen ist so nicht mehr nötig. Durch die Untergrabung der Legitimität der anderen Seite können deren politische Forderungen leichter zurückgewiesen werden, denn ein Eingehen auf diese würde in dieser Argumentationslogik nur den «Schurkenstaat» der LTTE im Nordosten stärken. Deshalb kann aus dieser Wahrnehmung eine Autonomie des Nordostens nicht befürwortet werden, solange die LTTE dort ihren «Terrorstaat» unterhält.

Der Hinweis auf den drohenden Terrorstaat im Nordosten ist auch ein Versuch der "Colombo-Elite», von gesamtstaatlichem Reformbedarf in verschiede- nen sozialen und politischen Feldern abzulenken: Die Unterentwicklung des singhalesisch bewohnten Südens wird zu einem nicht geringen Grad durch die Korruption und Vetternwirtschaft reproduziert, von der vor allem die englischsprachige Elite in Colombo und eine lokale, politische Elite, die sich auf Klientelbeziehungen stützt, profitieren. Dies begrenzt die Lebenschancen der ländlichen Jugendlichen im Süden Sri Lankas, die deshalb eine hohe Gewaltbereitschaft zeigen (MAYER 2002a: 26-29, 2002b; UYANGODA 2003: 47-51). Auch wird durch den Terrorismus- und Schurkenstaatsvorwurf verschleiert, dass die Demokratie in Sri Lanka nur noch formal besteht, aber durch korrupte Verwaltungs- und klientelistische Machtstrukturen zunehmend unterhöhlt wird (Foster 2003: 155; RaJasingham-SEnanayake 2003; Uyangoda 2000). Hinzu kommt, dass die Zentralregierung von 1977 bis 2002 unter dem «Prevention of Terrorismus Act (PTA)» regierte, der der Exekutive weitreichende Befugnisse einräumte. Unter dem Deckmantel des Bürgerkrieges und der Terrorismusbekämpfung kam es zu eklatanten Menschenrechtsverletzungen durch die Polizei und das Militär, wie z.B. Folter, Ermordung von Zivilisten (RösEl 1997: 333-335).

Aber auch außerhalb des Kriegsgebietes sind die demokratischen Grundlagen unterhöhlt: Formal werden weiterhin relativ freie Wahlen abgehalten, bei denen es auch zu Regierungswechseln kommt. In der Verfassungswirklichkeit ist jedoch ein Klientelsystem etabliert worden, das die Bevölkerung in Gewinner und Verlierer einteilt, je nachdem, ob man die «richtige» Partei unterstützt hat. Es kommt regelmäßig zu gewalttätigen politischen Auseinandersetzungen zwischen den Angehörigen verschiedener Parteien. Der bestehende Reformbedarf innerhalb der singhalesischen Gesellschaft und im srilankischen Staatswesen kann heruntergespielt werden, wenn man weiterhin auf die «Terroristen» im Nordosten und ihren potentiellen «Schurkenstaat» verweisen kann, den es zu verhindern gilt, um die eigene Sicherheit und Identität zu bewahren.

\subsection{Anker zur Identitätsbildung}

Im tamilischen Diskurs verschleiert die eindeutige Gegenüberstellung eines tamilischen Befreiungskampfes gegen einen singhalesischen «Schurkenstaat» bestehende Widersprüche innerhalb der tamilischen Gesellschaft. Die tamilische Gesellschaft ist geographisch, sozial und politisch in Klassen und Kasten fragmentiert: Die Schurkenbilder, die viele Tamilen im Nordosten von Singhalesen und ihrem Staat konstruieren, werden nicht notwendigerweise von Tamilen in Colombo geteilt. Insbesondere die tamilische Geschäftselite in Colombo hat wenig Verständnis für die Einstellungen und Aktionen der LTTE und nationalistischer Tamilen. Die staatliche Repression, 


\begin{tabular}{|c|c|c|c|}
\hline & «Singhalesischer» Diskurs & «Tamilischer» Diskurs & «Muslimischer» Diskurs \\
\hline $\begin{array}{l}\text { Schlüssel- } \\
\text { argument }\end{array}$ & $\begin{array}{l}\text { Die LTTE ist eine terroristische } \\
\text { Organisation, die einen Schurkenstaat im } \\
\text { Nordosten errichten möchte. }\end{array}$ & $\begin{array}{l}\text { Der singhalesische Staat ist ein } \\
\text { Schurkenstaat, der die tamilischen Bürger } \\
\text { diskriminiert und das tamilische Homeland } \\
\text { besetzt hält. }\end{array}$ & $\begin{array}{l}\text { Die LTTE schafft einen Quasi- } \\
\text { Schurkenstaat im Nordosten, der die } \\
\text { wirtschaftliche Prosperität der Muslime } \\
\text { gefährdet. }\end{array}$ \\
\hline $\begin{array}{l}\text { Diskursive } \\
\text { Argumente }\end{array}$ & $\begin{array}{l}\text { Die srilankische Regierung verbündet sich } \\
\text { weltweit mit demokratischen Regierungen } \\
\text { im Kampf gegen den Terrorismus. } \\
\text { - Eine demokratische Regierung kann nicht } \\
\text { mit Terroristen verhandeln, die einen } \\
\text { Schurkenstaat innerhalb des Territoriums } \\
\text { des srilankischen Rechtsstaates errichten } \\
\text { wollen. } \\
\text { - Tamil Eelam - die Bezeichnung für den } \\
\text { geforderten unabhängigen LTTE-Staat im } \\
\text { Nordosten von Sri Lanka - gilt als } \\
\text { Schurkenstaat, der die territoriale Integrität } \\
\text { der srilankischen Demokratie untergräbt. }\end{array}$ & $\begin{array}{l}\text { - Tamilen werden als zweitklassige Staats- } \\
\text { bürger behandelt und vom srilankischen } \\
\text { Staat unter den Generalverdacht des } \\
\text { Terrorismus gestellt. } \\
\text { - Ethnisch motivierte Kolonisationsprojekte } \\
\text { im Nordosten schüren bei den Tamilen die } \\
\text { Angst, zu einer «Minderheit in ihrem } \\
\text { eigenen Homeland» zu werden, womit ihre } \\
\text { Forderung nach Unabhängigkeit } \\
\text { unterwandert werden könnte. } \\
\text { - Die srilankische Armee und Polizei führen } \\
\text { sich im Nordosten nicht als «Sicherheits- } \\
\text { kräfte», sondern als Besatzungsarmee auf. }\end{array}$ & $\begin{array}{l}\text { - Die LTTE schafft einen Quasi- } \\
\text { Schurkenstaat im Nordosten, der von } \\
\text { Tamilen dominiert wird. Dieser Quasi-Staat } \\
\text { erhebt Steuern und Abgaben auch von } \\
\text { Muslimen und schüchtert ihre politischen } \\
\text { Vertreter ein. } \\
\text { - Die politische Verwundbarkeit der Muslime } \\
\text { steigt dadurch, insbesondere weil die } \\
\text { srilankische Armee die Muslime nicht vor } \\
\text { der LTTE schützen kann (und will?). } \\
\text { - Wegen der Errichtung dieses Quasi- } \\
\text { Schurkenstaates brauchen die Muslime } \\
\text { besondere Schutzmaßnahmen und ein } \\
\text { eigenes Homeland im Nordosten, } \\
\text { unabhängig von Tamil Eelam. }\end{array}$ \\
\hline $\begin{array}{l}\text { Verdeckte } \\
\text { Interessen }\end{array}$ & $\begin{array}{l}\text { Verweigert jeden Ansatzpunkt für } \\
\text { Verhandlungen mit den tamilischen } \\
\text { «Terroristen». } \\
\text { - Erlaubt der singhalesischen Elite, interne } \\
\text { soziale, ökonomische und politische } \\
\text { Widersprüche innerhalb der singhalesischen } \\
\text { Gesellschaft zu verdecken und von diesen } \\
\text { abzulenken. }\end{array}$ & $\begin{array}{l}\text { Verschleiert die politische Unterdrückung } \\
\text { und die autoritäre Herrschaft der LTTE im } \\
\text { Nordosten. } \\
\text { - Übersieht die hybriden, mehrdeutigen } \\
\text { Identitäten der Menschen und die internen } \\
\text { sozialen Widersprüche der tamilischen } \\
\text { Gesellschaft im Nordosten (z.B. } \\
\text { Kastenwesen, politische Opposition). }\end{array}$ & $\begin{array}{l}\text { - Versucht, die Kriegsgewinnler unter den } \\
\text { Muslimen gegen Regressforderungen } \\
\text { abzusichern. } \\
\text { - Erlaubt muslimischen Politikern, ihre } \\
\text { Wählerklientel an die konkurrierenden } \\
\text { muslimischen Parteien zu binden. }\end{array}$ \\
\hline
\end{tabular}

Tab. 1: Vergleich der Schurkenstaat-Diskurse in Sri Lanka

Comparison of rogue state discourse in Sri Lanka

Comparaison des discours d'Etat-voyou au Sri Lanka

Entwurf: B. KORF 
gerechtfertigt durch den Kampf gegen den Terrorismus, führte dazu, dass weite Teile der tamilischen Bevölkerung im kriegsversehrten Nordosten die LTTE und ihr autoritäres Regime während der letzten Jahre des Bürgerkrieges erduldet oder stillschweigend unterstützt haben. Die LTTE bestraft politischen Widerspruch, Oppositionelle werden eingeschüchtert und ermordet, illegale Steuern erhoben, ohne dass der Bevölkerung Rechenschaft darüber abgelegt würde, wofür diese Mittel verwendet werden (SARVANANTHAN 2002, 2003). Zudem führt die LTTE weiterhin Kinderrekrutierungen durch, um ihre Armee zu verstärken. Viele Tamilen im Nordosten wünschen sich jedoch kein autoritär regiertes Eelam.

Gerade in der tamilischen Diaspora - einer wichtigen Finanzquelle der LTTE - werden die Schurkenbilder des «ethnisch Anderen» und die Vorbehalte gegenüber dem srilankischen «Schurkenstaat» gepflegt und auch von der LTTE geschürt, um ihre Finanzierung zu sichern. Während des Bürgerkrieges sind viele wohlhabendere Tamilen nach Colombo (SidDharTan 2003: 308-310) und ins Ausland geflohen (Fuglerod 1999, 2003) und haben so eine einflussreiche TamilenDiaspora geschaffen. Das enge Netz transnationaler familiärer Beziehungen wird durch ein gut organisiertes System von Informationen und Propaganda politisch - auch über das Internet - einseitig auf die Belange von Tamil Eelam eingeschworen. Die tamilische «Nation» entsteht hier als Ikone, nicht als Platz zum Leben, sondern als etwas, an das man glauben kann und das eine vereinigende Ideologie bietet (Jeganathan 1998). Politische Forderungen aus der Diaspora sind daher oft radikaler und weniger kompromissbereit als diejenigen von Tamilen, die im Nordosten Sri Lankas leben. Tamilen, die außerhalb des Nordostens, aber innerhalb von Sri Lanka Zuflucht gefunden haben, vor allem in Colombo, müssen ihr «Tamilisch-Sein» («tamilness») im öffentlichen und privaten Raum neu aushandeln: Während der innere Zusammenhalt im privaten Raum unter den Tamilen durch das gemeinsame Leid verstärkt wurde, wird die tamilische Identität im öffentlichen Raum möglichst versteckt, um nicht unnötig Ärger mit den Sicherheitsorganen zu bekommen (SIDDHARTAN 2003: 317).

\subsection{Territorialisierung als Verhandlungsstrategie - ein muslimisches Homeland?}

In der muslimischen Gesellschaft hat sich in den letzten zwanzig Jahren mit dem Sri Lanka Muslim Congress (SLMC) eine Partei etabliert, die explizit die Interessen der Ostküstenmuslime vertritt (O'SULLIVAN 1999; WaGner 1990). Diese Partei hat in der nationalen Politik die Rolle des Züngleins an der Waage übernommen, das der jeweiligen singhalesischen Mehrheitspartei die absolute Mehrheit im Parlament sichert. Der SLMC hat sich deshalb von Anbeginn als kliente- listische und ethnische Partei verstanden und vor allem für Entwicklungsvorhaben in muslimischen Gebieten Lobbying betrieben. Auch gab es - als Reaktion auf die muslimisch-tamilischen Unruhen - verschiedene Versuche, eine Verwaltungsbezirksreform durchzusetzen, die zu ethnisch homogenen Einheiten führen sollten (McGilvray 2003; Thangarajah 2003: 33-34).

Allerdings sind die muslimischen Politiker seit einiger Zeit über den aus ihrer Sicht optimalen Kurs zerstritten und es ist zur Gründung neuer Parteien neben dem SLMC gekommen. Zur Unterstützung eines Interessensvertreters für die Ostküstenmuslime nutzen die konkurrierenden Parteien vielfach die Schurkenbilder und die Angst vor einem LTTE-Staat. Diese Angst nutzen einige muslimische Politiker, um die Legitimation für ein muslimisches Homeland zu konstruieren. Die Unruhen zwischen Tamilen und Muslimen geben ihnen dazu die Legitimität, denn sie zeigen die Verwundbarkeit der muslimischen Minderheit auf: Sollte die LTTE die volle Kontrolle über einen autonomen Nordosten erhalten, dann gäbe es keinen spezifischen Schutz für die muslimische Minderheit mehr, der jetzt noch durch die Präsenz von Armee und Polizei zumindest teilweise gegeben ist.

\subsection{Fiktionen ethnischer Reinheit und die Politik der Verwundbarkeit}

Schurkendiskurse bereiten die Legitimation für ethnisch reine Räume. Ethnische Reinheit ist jedoch eine Fiktion. In den multi-ethnischen Gebieten im Osten Sri Lankas haben die gewalttätigen Auseinandersetzungen der Vergangenheit eine besondere Sensibilität unter der lokalen Bevölkerung hinsichtlich ihrer jeweiligen ethnischen Minderheits- oder Mehrheitsposition im lokalen und regionalen Kontext erzeugt, denn dies determiniert die eigene politische und militärische Verwundbarkeit (WaTTS \& Bohle 2003; Korf 2003, 2004, 2005).

Minderheit oder Mehrheit zu sein ist erstens eine Frage des geographischen Maßstabes, wie sich an den Siedlungsmustern im Nordosten zeigt: Tamilen - auf nationaler Ebene eine Minderheit - sind in der Nordostprovinz die Mehrheit, Muslime stellen eine signifikante Minderheit dar, ebenso wie singhalesische Siedler. Im multi-ethnischen Osten stellen einzelne ethnische Gruppen regional die (relative) Mehrheit dar, sowohl auf Distrikt- als auch auf Unterdistriktebene.

Zweitens lösen sich Identitäten in der politischen Geographie der Gewalt und des Bürgerkrieges im jeweiligen Raum-Zeit-Kontext auf. Im Mikrokosmos des alltäglichen Zusammenlebens sind die Lebenssysteme von Muslimen und Tamilen im Osten voneinander abhängig, die Verwundbarkeit im Überleben ist kontextuell beeinflusst von der politischen Geographie 
der Gewalt. Ein Beispiel kann diese Dynamik verdeutlichen: In politisch angespannten Zeiten trauen sich tamilische Bauern in Muthur im Osten Sri Lankas nicht mehr, ihre landwirtschaftlichen Produkte in muslimisch bewohnten Städten zu vermarkten; muslimische Bauern zögern, ihre Felder zu bestellen, wenn sie auf dem Weg dorthin tamilische Dörfer passieren müssen oder wenn ihre Felder in Gebieten liegen, die mehrheitlich von Tamilen bearbeitet werden (KoRF 2003, 2005).

Drittens zeigen anthropologische Studien, dass das Konzept «ethnischer Reinheit» ein politisches Konstrukt des kolonialen und post-kolonialen Sri Lankas ist. Diese Arbeiten zeigen zum Beispiel die hybride Natur ethnischer Identität im Osten Sri Lankas. Die klare Trennung zwischen «tamilischer» und «muslimischer» Identität ist jüngeren Ursprungs und wurde vor allem durch den Bürgerkrieg dramatisch verschärft. Ursprünglich gab es jedoch viele verwandtschaftliche Bande zwischen tamilischen und muslimischen Familien (Fuglerod 2003; McGilvray 2003).

\section{Fazit}

Das Böse - der Schurke - wird gebraucht zur eigenen Reinigung. Mit Bezug auf die Politik der «Achse des Bösen» wies der Philosoph RÜdIger SAFRANSKI darauf hin, dass:

«die Illusion der Reinigung der Welt und der Gesellschaft durch eine ultimative, vollständige Ausscheidung des Bösen, zum Beispiel durch Eroberung von «Schurkenstaaten», selbst zu einem Mechanismus werden [kann], der die schlimmen Dinge erst produziert» (SAFRANSKI 2003: 73).

In Sri Lanka sollen die Schurkendiskurse den politischen Weg für eine solche «Reinigung» schaffen, indem «ethnische Minderheiten» quasi abgeschafft und Fiktionen ethnischer «Reinheit» geschaffen werden. Diese Reinigungspolitik hat jedoch die ethnische Gewalt zu verantworten.

Tamilen und Muslime argumentieren, dass der Konflikt dadurch überwunden werden soll, dass den ethnischen Minderheiten jeweils eigene quasi-staatliche Entitäten zugestanden werden - Homelands. Die LTTE versucht, ein muslimisches Homeland innerhalb ihres eigenen Homelands zu verhindern, weil sie in ihrem eigenen Machtraum unbeschränkt herrschen will. Muslimische Politiker wiederum haben in der Vergangenheit Praktiken eines ethnischen Klientelismus vorgeführt, die nur auf das ethnische «Selbst» gerichtet sind. Gegen diese Territorialisierung der Konfliktlösung wenden sich singhalesische Nationalisten, weil sie dadurch einen Verlust der Identität und Macht des buddhistischen Staates Sri Lanka befürchten. Allen drei Parteien geht es also darum, eine «ethnisch reine» Welt zu schaffen, entweder als ethnisch homogene Homelands oder in der Form des singhalesisch-buddhistischen Staates, der den dort lebenden Minderheiten keine Rechte zugesteht.

Eine räumliche Trennung, wie sie implizit durch die Forderungen nach Homelands zum Ausdruck kommt, ist praktisch nicht durchführbar, denn diese vermeintlichen Lösungen würden nur wieder neue Minderheiten in den dann geschaffenen territorialen Einheiten erzeugen. Es gibt keine einfache Einordnung in Minderheits- und Mehrheitsethnie. Aufgrund der durchmischten Siedlungsstruktur mit einem Mosaik tamilischer und muslimischer Dörfer und Kleinstädte an der Ostküste Sri Lankas ist die Bildung «ethnisch homogener» Verwaltungseinheiten unter rein praktischen Gesichtspunkten nicht durchführbar. Wenn hinter der «Territorialisierung» der Konfliktlösung der Gedanke steht, ethnisch möglichst homogene geographische Einheiten zu schaffen, wird die Verwundbarkeit der dann geschaffenen Minderheiten innerhalb dieser Einheiten noch verstärkt: In vermeintlich «ethnisch homogenen» Räumen ist dann kein Raum, um die Lebenswelten und Menschenrechte der dort lebenden Minderheiten zu sichern, da ihre Anwesenheit nicht in das Konzept ethnischer Homogenität und Reinheit passt.

\section{Literatur}

AGnew, J. (2001): Making Political Geography. London: Arnold Publishers.

Chомsкy, N. (2000): Rogue States: The Use of Force in World Affairs. - New York: Southend Press.

Dalby, S. (1991): Critical Geopolitics: Discourse, Difference, Dissent. - In: Environment and Planning D: Society and Space 9:261-283.

Derrida, J. (2003): Voyous. - Paris: Edition Galilee.

DodDS, K.J. \& J. SidAWAY (1994): Locating Critical Geopolitics. - In:Environment and Planning D: Society and Space 12: 515-524.

Foster, Y. (2003): Jaffna - The Context of Development in a War for Peace. - In: MAYER, M., RAJASINGAMSenanayake, D. \& Y. Thangarajah (Hrsg.): Building Local Capacities for Peace: Rethinking Conflict and Development in Sri Lanka. - Delhi: Macmillan Publishers: 152-166.

Foucault, M. (1972): L' ordre du discours. - Paris: Gallimard.

Fuglerod, O. (1999): Life on the Outside: The Tamil Diaspora and Long-distance Nationalism. - London: Pluto Press.

Fuglerod, O. (2003): Local Communities and State Ambitions in Eastern Sri Lanka. - In: MAYER, M., Rajasingam-Senanayake, D. \& Y. Thangarajah (Hrsg.): Building Local Capacities for Peace: Rethink- 
ing Conflict and Development in Sri Lanka. - Delhi: Macmillan Publishers: 65-79.

GoodhAND, J. \& N. Lewer (1999): Sri Lanka: NGOs and Peace Building in Complex Emergencies. - In: Third World Quarterly 20, 1: 69-87.

INDRAJIT, S. (2003): De-merging of the North-Eastern province: Sinhala professionals abroad pledge support to President. - In: The Island v. 13.6.2003: 3.

Jeganathan, P. (1998): eelam.com: Place, Nation, and Imagination in Cyberspace. - In: Public Culture 10, 3: 515-528.

Klare, M. (1995): Rogue States and Nuclear Outlaws: America's Search for a New Foreign Policy. - New York: Farrar, Straus \& Giroux Press.

Korf, B. (2003): Geographien der Gewalt: Handlungsorientierte geographische Bürgerkriegsforschung in politisch-ökonomischer Perspektive. - In: Geographische Zeitschrift 91, 1:24-39.

KorF, B. (2004): War, Livelihoods, and Vulnerability in Sri Lanka. - In: Development and Change 35, 2: 177-197.

Korf, B. (2005): Rethinking the Greed-Grievance Nexus: Property Rights and the Political Economy of War in Sri Lanka. - In: Journal of Peace Research 42, 2: 201-217.

LiTWAK, R.S. (2000): Rogue States and US Foreign Policy: Containment After the Cold War. - Washington D.C.: The Woodrow Wilson Center Press.

MAYER, M. (2002a): Jugendkonflikte und Entwicklungsplanung: Eine sozialgeographische Analyse der Lebenschancen Jugendlicher und der Potenziale dezentraler Planung in Sri Lanka. - Saarbrücken: Breitenbach.

MAYER, M. (2002b): Jugend, Verwundbarkeit und Soziale Diskriminierung. Lebenschancen und Konfliktpotentiale Ländlicher Jugendlicher in Sri Lanka. - In: Geographica Helvetica 57, 1: 19-33.

McGilvray, D.B. (2003): Tamil and Muslim Identities in the East. - In: Marga Journal 1, 1:79-116.

O'Sullivan, M. (1999): Conflict as a Catalyst: The Changing Politics of the Sri Lankan Muslims. - In: Gamage, S. \& I.B. Watson (Hrsg.): Conflict and Community in Contemporary Sri Lanka. - New Delhi: Sage Publications: 253-278.

Ó Tuathail, G. (1996): Critical Geopolitics. The Politics of Writing Global Space. - Minneapolis: University of Minnesota Press.

Ó Tuathall, G. (2002): Theorizing Practical Geopolitical Reasoning: The Case of the United States' Response to the War in Bosnia. - In: Political Geography 21, 5: 601-628.

Ó Tuathail, G. \& S. Dalby (eds) (1998): Re-Thinking Geopolitics. Towards a Critical Geopolitics. - London: Routlegde.

Rajasingham-Senanayake, D. (2003): The Economics of Peace. - In: Polity 1, 1: 15-20.

RösEL, J. (1997): Der Bürgerkrieg auf Sri Lanka. Der
Tamilenkonflikt: Aufstieg und Niedergang eines singhalesischen Staates. - Baden-Baden: Nomos.

RuBIN, B. (1999): «Schurkenstaaten»: Amerikas Selbstverständnis und seine Beziehungen zur Welt. - In: Internationale Politik 54, 6:5-14.

SafranSKI, R. (2003): «Wir müssen uns dem Bösen stellen». Interview mit RÜDIgER SAFRANSKI. - In: der blaue reiter 17: 70-77.

Sarvananthan, M. (2002): Tigers, Tages, and the Tamils. - In: Pravada 8, 1+2:11-12.

Sarvananthan, M. (2003): What Impedes Economic Revival in the North \& East Province of Sri Lanka? - Unpublished paper, International Centre for Ethnic Studies (ICES), Colombo.

SChriJVERS, J. (1997): Tamil-Muslim Violence, Gender and Ethnic Relations in Eastern Sri Lanka. - In: Nethra II, 3: 10-39.

Siddhartan, M. (2003): Negotiating «Tamilness»: A Case Study of Jaffna Tamil Migrants to Colombo since 1990. - In: Mayer, M., Rajasingam-Senanayake, D. \& Y. Thangarajah (Hrsg.): Building Local Capacities for Peace: Rethinking Conflict and Development in Sri Lanka. - Delhi: Macmillan Publishers: 305-20.

Tамвiaн, S. (1986): Sri Lanka: Ethnic Fratricide and the Dismantling of Democracy. - Chicago: Chicago University Press.

Thangarajah, Y. (2003): Ethnicisation of the Devolution Debate and the Militarization of Civil Society in North-Eastern Sri Lanka. - In: MAYER, M., RAJASINgam-Senanayake, D. \& Y. Thangarajah (Hrsg.): Building Local Capacities for Peace: Rethinking Conflict and Development in Sri Lanka. - Delhi: Macmillan Publishers: $15-36$.

THE ISLAND v. 9.5.2003: Lanka will soon be a sovereign shell, warns Kadirgama. - Colombo: 1,3.

THE IsLAND v. 9.5.2003: The choice: Sovereignty of the nation or the «Peace Process». - Editorial, Colombo: 10.

The Island v. 21.5.2003: Don't denigrate the Sri Lankan nation. - Editorial, Colombo: 8.

THE IsLAND v. 6.5.2003: Don't barter sovereign rights. Editorial, Colombo: 10.

THE ISLAND v. 13.6.2003: Warlord preparing for war again? - Editorial, Colombo: 10.

University Teachers for Human Rights - Jaffna (UTHR - J) (2003): Rewarding Tyranny: Undermining the Democratic Potential for Peace. - Special Report No. 17, Colombo: University Teachers for Human Rights - Jaffna.

Uyangoda, J. (2000): A State of Desire? Some Reflections on the Unreformability of Sri Lanka's Post-Colonial Polity. - In: Hetrige, S.T. \& M. MAyer (Hrsg.): Sri Lanka at Crossroads: Dilemmas and Prospects After 50 Years of Independence. - Delhi: Macmillan Publishers.

Uyangoda, J. (2003): Social Conflict, Radical Resistance and Projects of State Power in Southern Sri 
Lanka: The Case of JVP. - In: MAYER, M., RAJASINGAMSenanayake, D. \& Y. Thangarajah (Hrsg.): Building Local Capacities for Peace: Rethinking Conflict and Development in Sri Lanka. - Delhi: Macmillan Publishers: 37-64.

WAGNER, C. (1990): Die Muslime Sri Lankas. - Freiburg: Arnold-Bergstraesser-Institut.

WatTs, M. \& H.-G. Bohle (2003): Verwundbarkeit, Sicherheit und Globalisierung. - In: GebHardT, H., Reuber, P. \& G. Wolkersdorfer (Hrsg.): Kulturgeographie: Aktuelle Ansätze und Entwicklungen. Berlin, Heidelberg: Spektrum, Akademischer Verlag: 67-82.

\section{Zusammenfassung: Wer hat Angst vorm Schurken- staat? Macht/Raum-Diskurse in Sri Lanka}

Dieser Artikel analysiert den Gebrauch geopolitischer Diskurse zur Legitimierung politischer Machtansprüche an Raum und Territorien, wie sie in Sri Lanka seit dem Ende des ethnischen Bürgerkrieges zum Tragen kommen. Die Analogie der «Schurken»-Metapher dient als analytischer Rahmen zur Untersuchung nationalistischer Diskurse innerhalb der drei ethnischen Gruppen in Sri Lanka, den Singhalesen, Tamilen und Muslimen. In diesen Diskursen geht es um konkurrierende Territorialansprüche zwischen den ethnischen Gruppen. Diese Diskurse versuchen, die eigenen Ansprüche argumentativ zu legitimieren und diejenigen des «ethnisch Anderen» zu unterminieren. Gelingt es, die andere Verhandlungspartei als «Schurken» darzustellen, lassen sich Strategien der Stärke und Gewalt besser rechtfertigen und Konfliktlösungen auf territoriale Argumente reduzieren. Diese «Schurken»-Logik konstruiert die Fiktion ethnisch homogener Räume und verdeckt dabei die Existenz von komplexen, multi-ethnischen Siedlungsstrukturen in den umstrittenen Gebieten im Nordosten einerseits und die relative Dynamik von Minderheits- und Mehrheitsstatus einer ethnischen Gruppe im jeweiligen lokalen Kontext andererseits.

\section{Summary: Who is afraid of the rogue state?} Discourse on power and spatial relations in Sri Lanka This article analyzes how ethnicity constructions go hand in hand with discourses on space and territories. In the case of Sri Lanka's ethnic conflict, these discourses create a social construct of the "(ethnic) other" as «rogue». Since contested claims for territories and statehood are at the core of the Sri Lankan political struggle, legitimizing ones own and discarding, delegit- imizing those claims of the «ethnic other» are essential strategies of nationalist groups within each ethnic constituency. Defining the "other party» in the Sri Lankan civil war as a «rogue» helps political actors to justify punitive policies based on force and violence rather than dialogue. The logic of «rogue» discourses creates a fiction of ethnic homogeneity and covers the complexity of multi-ethnic settlement patterns and the related fluidity of minority or majority status, especially in the multi-ethnic east of Sri Lanka.

\section{Résumé: Qui craint l'Etat-voyou? Les discours «pouvoir - espace» au Sri Lanka}

La présente contribution analyse le recours aux discours géopolitiques visant à légitimer des revendications de pouvoir en matière d'espace et de territoire, comme c'est le cas au Sri Lanka depuis la fin de la guerre civile, d'essence ethnique. L'analogie de la métaphore de voyous sert de cadre analytique à l'étude des discours nationalistes au sein des trois groupes ethniques du Sri Lanka: les Cingalais, les Tamouls et les Musulmans. Ces discours traduisent des revendications territoriales opposées. Argumentation à l'appui, ils tentent de légitimer leurs propres revendications et de miner celles de l'«ethniquement autre» des autres ethnies. Si le parti en négociation peut être présenté comme voyou, les stratégies de puissance et de violence se justifient plus facilement et les résolutions de conflits sont susceptibles d'être réduites à des arguments territoriaux. Cette logique de «voyous» construit une fiction présentant des espaces ethniques identiques, cachant ainsi, d'une part, les structures complexes d'habitations multi-ethniques dans les territoires controversés du nord-est, d'autre part, la relative dynamique du statut de minorité et de majorité des groupes ethniques dans leurs contextes locaux respectifs.

Dr. Benedikt Korf, Department of Geography, University of Liverpool, Liverpool, L69 3BX, United Kingdom.

e-mail: b.korf@liverpool.ac.uk

\section{Manuskripteingang/received/manuscrit entré le 20.5.2004}

Annahme zum Druck/accepted for publication/accepté pour l'impression: 9.6.2005 